\title{
TÉCNICAS EXPEDITAS NA CARACTERIZAÇÃO FÍSICA DA CORTIÇA DESTINADA À PRODUÇÃO DE ROLHA
}

\author{
Catarina Martins Morais ${ }^{1}$, Isabel Tinoco ${ }^{2}$, Miguel Pestana ${ }^{3}$, João Paulo Borges ${ }^{4}$ \\ ${ }^{1}$ Eng $^{a}$ de Materiais, M.Sc., Faculdade de Ciências e Tecnologia, UNL, Caparica, Portugal - catarinammorais@gmail.com \\ ${ }^{2}$ Eng ${ }^{a}$ Silvicultora, Instituto Nacional de Recursos Biológicos, Oeiras, Portugal - isabel.tinoco@efn.com.pt \\ ${ }^{3}$ Investigador Auxiliar, Ph.D., Instituto Nacional de Recursos Biológicos, Oeiras, Portugal - miguel.pestana@efn.com.pt \\ ${ }^{4}$ Professor Auxiliar, Ph.D., Faculdade de Ciências e Tecnologia, UNL, Caparica, Portugal - jpb @ fct.unl.pt \\ Recebido para publicação: 09/12/2009 - Aceito para publicação: 22/04/2010
}

\begin{abstract}
Resumo
Este trabalho pretende dar a conhecer a importância da análise de imagem e do ensaio de torção na caracterização física da cortiça para a produção de rolha, de modo expedito. As amostras foram preparadas para determinação do calibre, massa específica, umidade, porosidade, força e respectivo ângulo de torção. Procedeu-se à comparação da classificação qualitativa das cortiças segundo os critérios de avaliação visual e objetivo (características físicas). Os resultados deste trabalho permitem demonstrar: As cortiças utilizadas para a produção de rolha classificadas de muito boas apresentam baixa porosidade, força e respectivo ângulo elevados; As cortiças não usadas para rolha são de pior qualidade, apresentando valores elevados dos parâmetros de análise de imagem, não permitindo o fabrico de rolhas; Algumas cortiças que não têm características visuais apropriadas para a produção de rolha foram, no entanto, destinados para esse fim, pelo critério objetivo; As amostras que permitem a obtenção de rolhas apresentam uma relação inversamente proporcional entre o ângulo, a área mínima de poros e a força com o número de poros, a área máxima de poros e porosidade; Comparando os resultados com os obtidos por Pestana da Silva (2003), pode-se verificar que permitem uma triagem eficaz entre cortiças de boa e má qualidade.
\end{abstract}

Palavras-chave: Qualidade; cortiça; rolha; características físicas; análise multivariada.

\begin{abstract}
Usefulness of image analysis and torsion test as mechanical prompt in cork characterization intended to stopper production. The present work aims to contribute for a better knowledge about the importance of image analysis and twisting test in the characterization of cork to stopper production. The samples were prepared for obtaining quantitative data on the calibre, apparent density, humidity, porosity, rupture strength and its angle. The classification resulting from this data was compared with the classification according the visual evaluating. The results of this study allow emphasizing that: The corks used for stoppers classified as very good, have low porosity with a high rupture strength and angle; The corks not used for stoppers with lower quality, have high values of image analysis, not allowing the manufacture of stoppers; Some samples don't have visual characteristics suitable for the production of stoppers, however they were used to its production, according to the objective criterion; The planks that allow the stopper production, exhibit a relationship between the angle, the minimum size of pores and strain with the number of pores, the maximum area of pores and porosity, they act in inverse way; Comparing the results with those obtained by Pestana da Silva (2003), it was observed that these parameters allow an effective screening of cork qualification.

Keywords: Quality; cork; stopper; physical characteristics; multivariate analysis.
\end{abstract}

\section{INTRODUÇÃO}

A "avaliação da qualidade" é entendida como a definição de objetivos quantificados e pela existência de processos para a sua avaliação.

É um fato que a fileira da cortiça avalia a qualidade de forma diferente, a montante na cortiça no mato, e a jusante na rolha de cortiça natural. 
Assim, para as diferentes formas de caracterização da cortiça e tendo em conta os dois estrangulamentos da fileira - a produção florestal/indústria preparadora e a indústria rolheira/empresas engarrafadoras -, o nosso estudo foca-se no primeiro, já que o grau de especificações no segundo é muito maior, dado que o mercado utilizador final tem a preocupação de otimizar a satisfação dos seus clientes.

Com esse intuito e tendo como base Pestana da Silva (2003), que elaborou um estudo de caracterização da qualidade da cortiça para a produção de rolha, efetuou-se este trabalho com o objetivo de validar a metodologia expedita, reprodutível e independente do avaliador. Para tal, recorreu-se à aplicação de metodologias de análise multivariada, com vista à validação do critério objetivo de classificação de cortiças proposto por Pestana da Silva (2003).

\section{MATERIAL E MÉTODOS}

\section{Material}

Para se realizar o estudo proposto, foram escolhidas 35 árvores. As árvores escolhidas encontram-se em duas herdades situadas numa zona de grande produção de cortiça do município de Coruche.

$\mathrm{Na}$ Herdade do Caldeiril foi seleccionada uma única parcela, constituída por 10 árvores, e na Herdade do Vale da Cilha foram selecionadas 5 parcelas, cada uma constituída por 5 árvores.

A identificação das amostras foi feita por letras e números romanos, sendo que a primeira letra corresponde à herdade em estudo (C - Herdade do Caldeiril; V - Herdade do Vale da Cilha), o número identifica a árvore (número em romano) e a letra seguinte identifica a altura da despela (descortiçamento) - A, B e C são as diferentes alturas a que foram feitas as despelas, 0,40, 0,80 e 1,20 m, respectivamente. Exemplo:

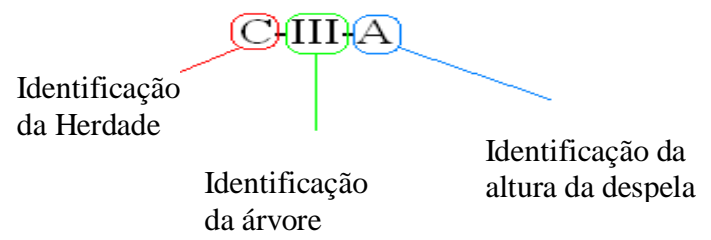

As pranchas extraídas das árvores foram cozidas e cortadas em amostras quadradas, de dimensões $10 \times 10 \mathrm{~cm}$, facilitando assim o seu manuseio.

Secionaram-se essas amostras para que fossem brocadas, obtendo-se dessa forma rolhas, que foram sujeitas a ensaios de torção.

Na Herdade do Caldeiril, do conjunto de 29 amostras brocadas, obtiveram-se 84 rolhas, e não as 87 que estavam previstas ( 3 rolhas por amostra).

Na Herdade do Vale da Cilha, do conjunto de 37 amostras brocadas, obtiveram-se 107 rolhas, e não as 111 previstas.

Esse fato resulta da limitação do calibre das amostras de não possibilitar a brocagem dessas cortiças para a obtenção de rolhas, motivo pelo qual não existem rolhas das amostras C-II-A, C-II-B, CVIII-C, V-V-B, V-XX-A e V-VII-A.

\section{Métodos}

As amostras foram cozidas durante uma hora, com o intuito de melhorar as suas capacidades mecânicas. Posteriormente, foram retiradas e colocadas num local arejado, onde foram prensadas. Essa operação destina-se a endireitar as cortiças, de modo que os procedimentos posteriores sejam mais exequíveis.

As cortiças não devem ficar exageradamente secas, pois a histerese do material leva a que as dimensões iniciais não sejam as mesmas depois de este ser seco e sujeito a umidificação.

Mediu-se o calibre das pranchas com um paquímetro normal e com uma incerteza de 0,02 $\mathrm{mm}$. As medições foram executadas nos dois extremos das pranchas. O valor do calibre é obtido pela média das duas determinações.

Segundo a Norma NP298 (ISO/DIS 1216), as cortiças são classificadas quanto ao calibre conforme indicado na tabela 1. 
Para o fabrico de rolhas, o calibre é uma característica relevante. Sendo assim, classificam-se pranchas com bom calibre aquelas que possibilitam a brocagem para obtenção de rolhas.

Para se efetuar a medição da umidade, utilizou-se o equipamento Aqua-Boy, usado na indústria da cortiça, constituído por uma sonda com cinco agulhas de $5 \mathrm{~cm}$ de comprimento cada. Para cada amostra foi efetuada uma leitura média obtida pelo resultado de 5 leituras.

Tabela 1. Classes de calibres das pranchas de cortiça e seus principais produtos.

Table 1. Class sizes of planks and its main products.

\begin{tabular}{lcc}
\hline Designação & Limite de espessura $(\mathbf{m m})$ & Principais produtos \\
\hline Delgadinha & $9-22$ & Discos \\
Delgada & $22-27$ & Discos \\
Meia-marca & $27-32$ & Rolhas \\
Marca & $32-40$ & Rolhas \\
Grossa & $40-54$ & Rolhas \\
Triângulo & $>54$ & Granulados \\
\hline
\end{tabular}

A cortiça foi agrupada quanto à umidade, de modo a garantir que as amostras possam ter o mesmo comportamento nos ensaios mecânicos.

Após essa medição, as amostras foram lixadas para regularizar a superfície e sujeitas a um jato de ar comprimido, de modo a limpar a superfície, para que se consiga obter uma imagem nítida e com os poros perfeitamente delimitados.

O estudo da porosidade fez-se através da análise de imagem com uma câmara digital CCD FOculus IEEE 1394com 6 Mega Pixels, com o programa da COGNEX Vision Pró 4 CR 2.

A cortiça pode ser classificada, quanto à porosidade, segundo a tabela 2 (FORTES et al., 2004; PESTANA et al., 2005).

Tabela 2. Diferenciação da porosidade das pranchas de cortiça e sua respectiva qualidade.

Table 2. Differentiation of cork planks porosity and its quality.

\begin{tabular}{lcc}
\hline Designação & Limite de porosidade & Classe de qualidade \\
\hline Pouco porosa & $<2 \%$ & Boa \\
Mediana & $2-4 \%$ & Razoável \\
Muito porosa & $>4 \%$ & Má \\
\hline
\end{tabular}

Além da porosidade, determinou-se também o número de poros $/ \mathrm{cm}^{2}$. Esse parâmetro permite obter um outro resultado, que pode ser comparado com o da porosidade.

As amostras utilizadas na análise de imagem foram secionadas e brocadas numa brocadora manual.

Foi determinada a massa específica (dap) das rolhas através da fórmula:

Em que: $m$ - massa da rolha;

$$
d_{a p}=\frac{m}{\pi \times r^{2} \times h}
$$

$r$ - raio médio da rolha;

$h$ - altura da rolha.

A pesagem das rolhas foi efetuada numa balança com incerteza de $0,5 \mathrm{mg}$ e uma precisão de $1 \mathrm{mg}$, obtendo-se assim a massa.

Para o cálculo do volume, mediram-se a altura e dois raios da base perpendicularmente um ao outro. Para tal, utilizou-se um paquímetro, com uma incerteza de $0,02 \mathrm{~mm}$, acertado a zero. A rolha é colocada no centro das hastes do paquímetro, registrando-se o valor amostrado. A medição dos diâmetros das rolhas é efetuada num topo, em duas posições perpendiculares.

Para a execução do ensaio de torção, recorreu-se à metodologia aplicada para a cortiça na norma NP 2803-6, onde esse ensaio faz parte da caracterização físico-mecânica da rolha cilíndrica de aglomerado composto de cortiça (PESTANA et al., 2006). 
O ensaio de torção efetuou-se em rolhas retiradas de amostras cujo calibre era igual ou maior a $25 \mathrm{~mm}$ de espessura. A broca usada nessa operação tem um diâmetro externo de $27 \mathrm{~mm}$ e um interno de $23 \mathrm{~mm}$, e uma altura que atravessa a amostra seccionada no sentido perpendicular aos canais lenticulares, de maneira a obter uma rolha com uma altura próxima de $50 \mathrm{~mm}$ (PESTANA et al., 2006).

Através da realização do ensaio, o sistema de aquisição de dados permitiu-nos obter os valores da força e do ângulo (PESTANA et al., 2006).

\section{Tratamento dos dados experimentais}

A caracterização das amostras foi auxiliada através da determinação dos valores médios da força, ângulo e massa específica das rolhas. Tal procedimento deve-se ao fato de ser impossível determinar rigorosamente esses parâmetros para as amostras, permitindo assim obter os valores médios dessas características identificadoras das propriedades de um material anisotrópico.

Utilizou-se a análise de taxonomia numérica como ferramenta para o tratamento dos dados experimentais.

Assim, para cada um dos conjuntos de dados, preparou-se previamente uma matriz de dados, com número de linhas (Operational Taxonomic Units - OTUs) correspondente ao objeto em análise (amostras) e com número de colunas correspondente às variáveis a analisar (PESTANA DA SILVA, 2003).

Dada a natureza diversa das diferentes variáveis procedeu-se à estandardização da matriz original, obtendo-se uma nova matriz de dados estandardizados, em que a média de valores de cada característica é agora zero e a respectiva variância 1. Nessa operação, calcula-se para cada característica a respectiva média e desvio padrão, e, em seguida, substitui-se cada valor original pelo quociente entre a sua diferença à média e o respectivo desvio padrão (PESTANA DA SILVA, 2003).

Calculou-se a semelhança, utilizando como coeficiente a distância euclidiana média.

Esse coeficiente representa a distância entre os pontos representativos de duas amostras num espaço, que terá tantas dimensões quantas as características utilizadas. Para o caso de objetos em análises iguais, essa distância é zero, e aumenta com a dessemelhança entre eles (PESTANA DA SILVA, 2003).

Dos vários métodos de agregação do tipo sequencial, aglomerativo, hierárquico e de não sobreposição, i. e., do tipo designado por SAHN (SNEATH; SOKAL, 1973), usou-se o método Unweighted Pair - Group Method Using Arithmetic Averages (UPGMA) (PESTANA DA SILVA, 2003).

Os resultados assim obtidos são apresentados sob a forma de uma estrutura ramificada, em que os diferentes ramos se relacionam de acordo com os valores das medidas de semelhança ou dessemelhança em que se baseou o método de agregação, que se designa por fenograma (PESTANA DA SILVA, 2003).

Para este fenograma, foi calculado o coeficiente de correlação cofenética (SOKAL; ROHLF, 1962) entre a matriz de valores cofenéticos, que exprimem a relação de semelhança entre OTUs implícita no fenograma, e a matriz de semelhança (ou dessemelhança). Esse coeficiente de correlação cofenética indica o grau de concordância entre as duas matrizes, permitindo avaliar se o fenograma é uma representação aceitável daquelas distâncias (PESTANA DA SILVA, 2003).

Usou-se, ainda, um outro método de agregação, para melhor entendimento dos resultados: o método de agregação designado árvore de conexão mínima (MST - Mininum Spanning tree).

Para obtenção da representação gráfica dos objetos a estudar ao longo dos eixos, num número reduzido de dimensões, geralmente duas ou três, preservando o máximo de variabilidade da matriz multidimensional dos dados originais, selecionou-se o método de ordenação em componentes principais (REIS, 2001).

Foram feitas as projeções das variáveis que caracterizam as entidades em estudo nas duas primeiras componentes principais, o que permite analisar a contribuição de cada uma na disposição espacial dos objetos em estudo.

Para esses cálculos, recorreu-se a um sistema de programas NTSYSPC (VER. 2.1) (ROHLF, 1997).

\section{RESULTADOS E DISCUSSÃO}

\section{Classificação prévia}

Inicialmente a cortiça em estudo foi submetida a uma classificação por inspeção visual, tendo como critério o aspecto. A classificação foi feita por uma associação florestal, tendo como características de 
estudo: espessura de pré-cozedura, espessura pós-cozedura, espessura média, aumento percentual após cozedura e o número de linhas para as duas herdades. O número de linhas (uma linha equivale a 2,256 $\mathrm{mm}$ ) da cortiça permite classificá-la pela espessura, conhecendo-se assim qual é o seu destino (produto final) tabela 3 e tabela 4, respectivamente, para a Herdade do Caldeiril e Herdade do Vale da Cilha.

As amostras foram classificadas de 1 a 7 , em que a classificação 1 corresponde a qualidade superior e classificação 7 corresponde a pior qualidade.

Herdade do Caldeiril

Através do estudo da tabela 3, pode-se dizer que a percentagem de cortiça rolhável na Herdade do Caldeiril será $80,00 \%$ (soma dos valores das células a verde escuro), a percentagem de cortiça delgada será $16,67 \%$ (soma dos valores das células a verde claro) e a de cortiça para granular 3,33\%.

Herdade do Vale da Cilha

Através do estudo da tabela 4, pode-se dizer que a percentagem de cortiça rolhável na Herdade do Vale da Cilha será 33,33\% (soma dos valores das células a verde escuro), a percentagem de cortiça delgada será $41,33 \%$ (soma dos valores das células a verde claro) e a de cortiça para granular $25,33 \%$.

Tabela 3. Distribuição percentual da amostra por calibres e qualidades de cortiça proveniente da Herdade do Caldeiril.

Table 3. Percentage distribution of the sample by calibre and cork qualities from the Herdade do Caldeiril.

\begin{tabular}{|c|c|c|c|c|c|}
\hline \multirow{2}{*}{ Calibres } & & \multicolumn{4}{|c|}{ Qualidade \% } \\
\hline & & $1^{\mathrm{a}} \mathrm{a} 3^{\mathrm{a}}$ & $4^{a}$ e $5^{a}$ & $6^{\mathbf{a}}$ & Refugo \\
\hline Delgadinha & 6 a 8 linhas & 0,00 & & & \\
\hline Delgadinha & 8 a 10 linhas & 0,00 & 0,00 & 0,00 & \\
\hline Delgada & 10 a 12 linhas & \multicolumn{2}{|c|}{10,00} & 6,67 & \\
\hline Marca e meia-marca & 12 a 18 linhas & \multicolumn{2}{|c|}{53,33} & 6,67 & 3,33 \\
\hline Grossa & $>18$ linhas & \multicolumn{3}{|c|}{20,00} & \\
\hline
\end{tabular}

Tabela 4. Distribuição percentual da amostra por calibre e qualidade das cortiças provenientes da Herdade do Vale da Cilha.

Table 4. Percentage distribution of the sample by calibre and cork qualities from the Herdade do Vale da Cilha.

\begin{tabular}{|c|c|c|c|c|c|}
\hline \multirow{2}{*}{ Calibres } & & \multicolumn{4}{|c|}{ Qualidade \% } \\
\hline & & $1^{a} a^{a}$ & $4^{a}$ e $5^{a}$ & $6^{\mathbf{a}}$ & Refugo \\
\hline Delgadinha & 6 a 8 linhas & 0,00 & & & \\
\hline Delgadinha & 8 a 10 linhas & 1,33 & 6,67 & 1,33 & \\
\hline Delgada & 10 a 12 linhas & & & 4,00 & \\
\hline Marca e meia-marca & 12 a 18 linhas & & & 5,33 & 25,33 \\
\hline Grossa & $>18$ linhas & & $1,33 \%$ & & \\
\hline
\end{tabular}

\section{Análise das amostras de cortiça}

Os dados dos ensaios físicos, força e ângulo de torção, utilizados para o cálculo do binário máximo e tensão de corte no ensaio de torção, são usados diretamente, visto que essa metodologia de tratamento de dados não permite usar variáveis que sejam combinações de outras, mas sempre características realmente medidas em cada objeto de estudo, o que não acontece com o binário e a tensão de corte.

Análise das amostras por métodos de taxonomia numérica da cortiça rolhável

Para realizar essa análise, foi necessário recorrer a uma matriz de dados composta por 66 linhas, correspondentes às 66 amostras estudadas, e por 9 colunas, referentes às características físicas e de análise de imagem.

FLORESTA, Curitiba, PR, v. 40, n. 4, p. 889-899, out./dez. 2010. 
O fenograma (Figura 1) obtido a partir da matriz de distâncias, utilizando o método UPGMA, representa de forma adequada a respectiva matriz, por possuir um coeficiente de correlação cofenética ( $\mathrm{r}$ ) de 0,862 , sendo o fenograma uma representação aceitável das distâncias.

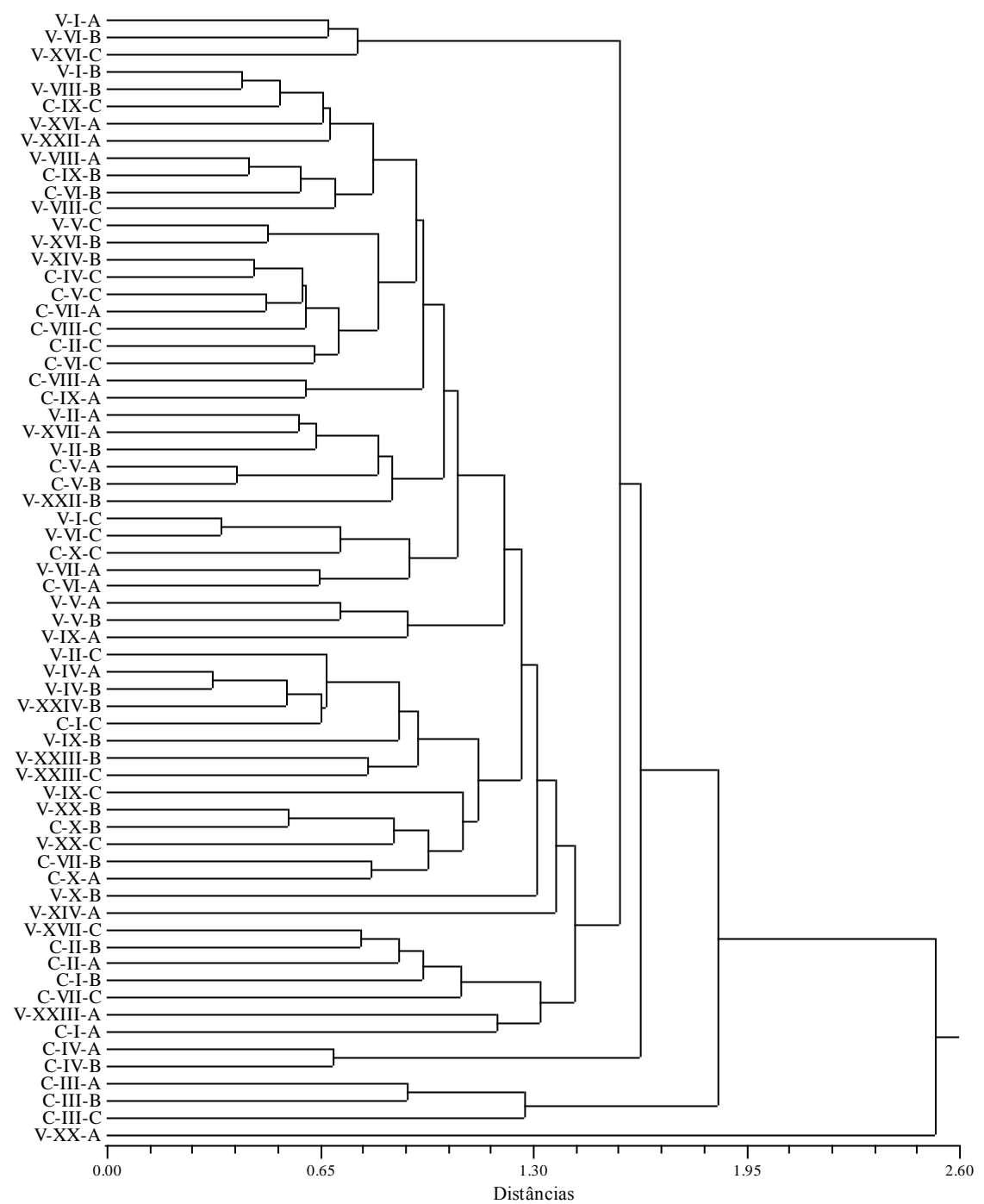

Figura 1. Fenograma de distância das 66 amostras, baseado no método UPGMA, aplicado à matriz distâncias $(r=0,862)$.

Figure 1. Phenogram distance of 66 samples, based on the UPGMA method, applied to matrix distances $(r=0,862)$.

Pela observação do fenograma, é possível visualizar dois grupos aos quais se liga um outlier, VXX-A. O primeiro grupo é constituído pela amostra C-III-C e pelos subgrupos C-III-B e C-III-A. O segundo grupo é constituído pelo subgrupo C-IV-B e C-IV-A, pelo subgrupo V-I-A, V-VI-B e V-XVI-C e pelas amostras restantes, cujo conjunto representa outro subgrupo. Em resumo, os diferentes grupos de amostras que se observam no fenograma revelam uma lógica para essa ligação, pois, com poucas exceções, são provenientes da mesma árvore e zona de despela.

Comparando-se a disposição espacial das amostras resultante da análise em componentes principais (Figura 2) com a agregação obtida no fenograma de distâncias, verifica-se uma grande concordância entre os dois resultados. 
Ao observar-se a figura 3 em conjunto com a tabela 5, pode-se dizer que a primeira componente principal influencia o número de poros, a área máxima de poros, a área mínima de poros e a porosidade. As variáveis influenciadas pela segunda componente principal são a massa específica, a umidade e o calibre, sendo a força de torção e o ângulo de torção influenciados pela terceira componente principal.

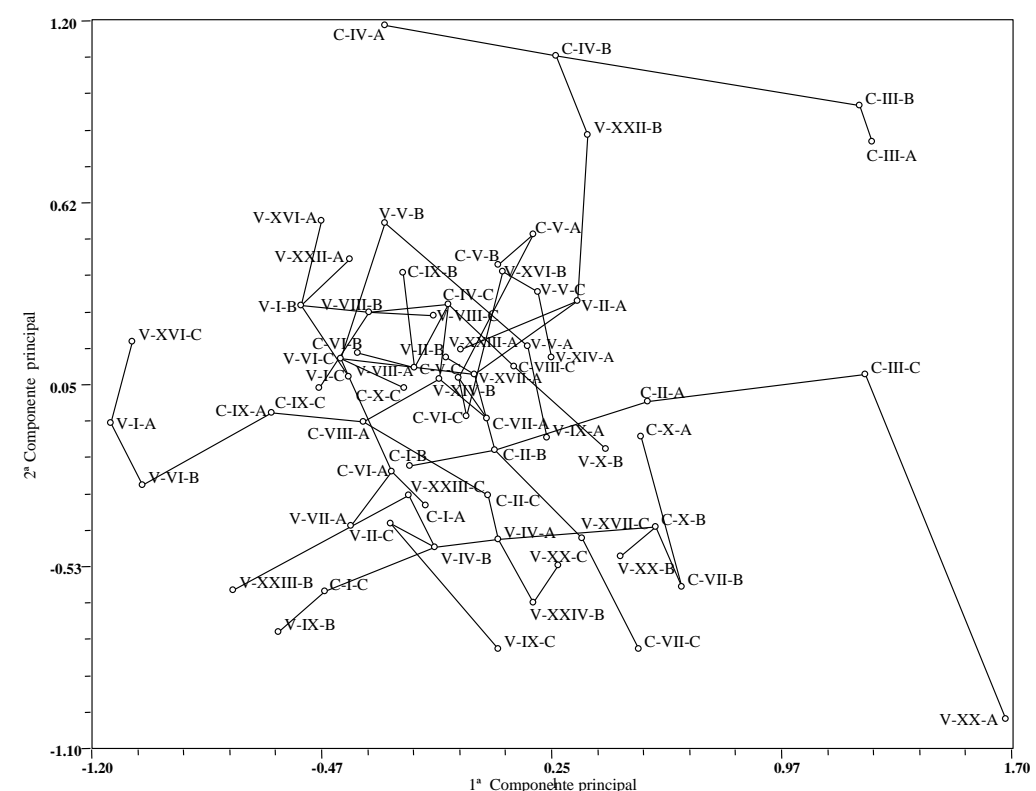

Figura 2. Projeção de 66 amostras no plano definido pelas duas primeiras componentes principais, a que foi sobreposta a árvore de conexão mínima para a detecção de distorções locais.

Figure 2. Projection of 66 samples in the plane of the first two principal components, which was superimposed on the minimum spanning tree to the detection of local distortions.

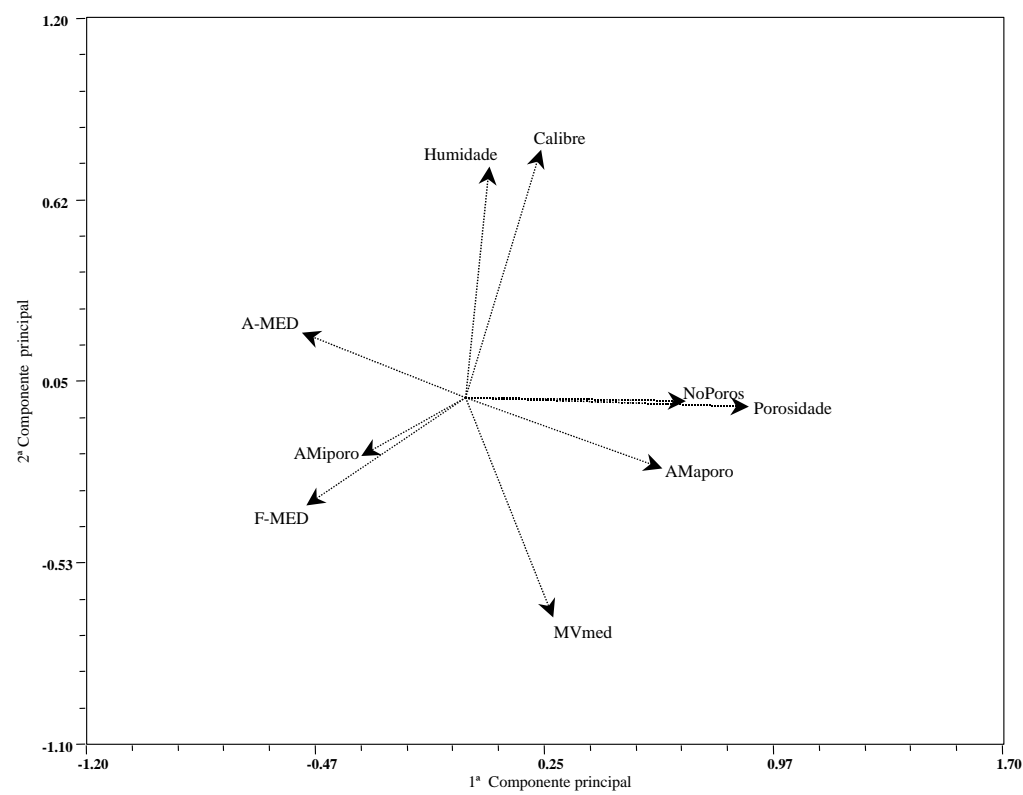

Figura 3. Projeções das variáveis físicas e de análise de imagem das amostras no plano definido pelas duas primeiras componentes principais.

Figure 3. Projections of the physical variables and image analysis from samples in the plane of the first two principal components.

FLORESTA, Curitiba, PR, v. 40, n. 4, p. 889-899, out./dez. 2010. 
Tabela 5. Correlação entre as variáveis originais e as três primeiras componentes principais.

Table 5. Correlation between the original variables and the first three principal components.

\begin{tabular}{lccc}
\hline \multirow{2}{*}{ Variáveis } & \multicolumn{3}{c}{ Componentes principais } \\
\cline { 2 - 4 } & $\mathbf{1}$ & $\mathbf{2}$ & $\mathbf{3}$ \\
\hline Umidade & 0.0770 & 0.7321 & 0.2774 \\
Calibre & 0.2394 & 0.7837 & -0.0256 \\
Noporos & 0.6961 & -0.0137 & -0.1680 \\
AMaporo & 0.6218 & -0.2265 & 0.5919 \\
AMiporo & -0.3283 & -0.1866 & -0.1018 \\
Porosidade & 0.8978 & -0.0295 & 0.3202 \\
MVmed & 0.2786 & -0.6995 & -0.0285 \\
F-MED & -0.5038 & -0.3433 & 0.5709 \\
A-MED & -0.5190 & 0.2050 & 0.5620 \\
\hline
\end{tabular}

Os dois eixos principais em conjunto representam $59,45 \%$ da variância total.

Análise das amostras por métodos de taxonomia numérica da cortiça não rolhável

Para realizar essa análise, foi necessário recorrer a uma matriz de dados composta por 36 linhas, correspondentes às 36 amostras estudadas, e por 6 colunas, referentes às características físicas e de análise de imagem.

O fenograma de distâncias (Figura 4) obtido a partir da matriz de distâncias, utilizando o método UPGMA, representa de forma adequada a respectiva matriz, por possuir um coeficiente de correlação cofenética (r) de 0,798, o que indicia uma representação aceitável das distâncias.

Pela observação do fenograma, é possível visualizar três grupos aos quais se ligam dois outliers (V-XII-C e V-XXIV-C). Um dos grupos é constituído pelas amostras V-III-A, V-IV-C e V-XXIV-A. Outro grupo é constituído pelas amostras V-XI-A, V-XV-A, V-XV-B, V-XXI-B, V-XXV-C, V-XV-C, V$\mathrm{XXV}-\mathrm{A}$ e V-XI-C. As amostras restantes constituem o terceiro grupo, que determina subgrupos no seu interior, como são os casos das amostras V-XIII-A e V-XVIII-C. Essas duas amostras agregam-se à amostra V-XIII-C, e a esse conjunto de três amostras agrega-se ainda a amostra V-XIII-B. Finalmente, a esse conjunto de quatro amostras agrega-se a amostra V-VI-A. Em resumo, os diferentes grupos de amostras que se observam no fenograma revelam uma lógica para essa ligação, pois, com poucas excepções, são provenientes da mesma árvore e zona de despela.

Comparando a disposição espacial das amostras resultante da análise em componentes principais (Figura 5), com a agregação obtida no fenograma de distâncias, verifica-se uma grande concordância entre os dois resultados.

Ao observar-se a figura 6 em conjunto com a tabela 6 , pode-se dizer que a primeira componente principal influencia o número de poros, a área máxima de poros e a porosidade. As variáveis influenciadas pela segunda componente principal são a umidade e o calibre. A terceira componente é controlada pela área mínima de poros.

Tabela 6. Correlação entre as variáveis originais e as três primeiras componentes principais.

Table 6. Correlation between the original variables and the first three principal components.

\begin{tabular}{lccc}
\hline \multirow{2}{*}{ Variáveis } & \multicolumn{3}{c}{ Componentes principais } \\
\cline { 2 - 4 } & 1 & 2 & 3 \\
\hline umidade & 0.4094 & 0.6046 & 0.4073 \\
Calibre & 0.5467 & 0.5733 & 0.2450 \\
Noporo & -0.6491 & 0.3828 & 0.1281 \\
AMAporo & -0.8328 & 0.1521 & 0.0503 \\
AMiporo & 0.0171 & -0.6242 & 0.7754 \\
Porosidade & -0.9024 & 0.1941 & 0.2093 \\
\hline
\end{tabular}

Os dois eixos principais em conjunto representam $71,95 \%$ da variância total. 


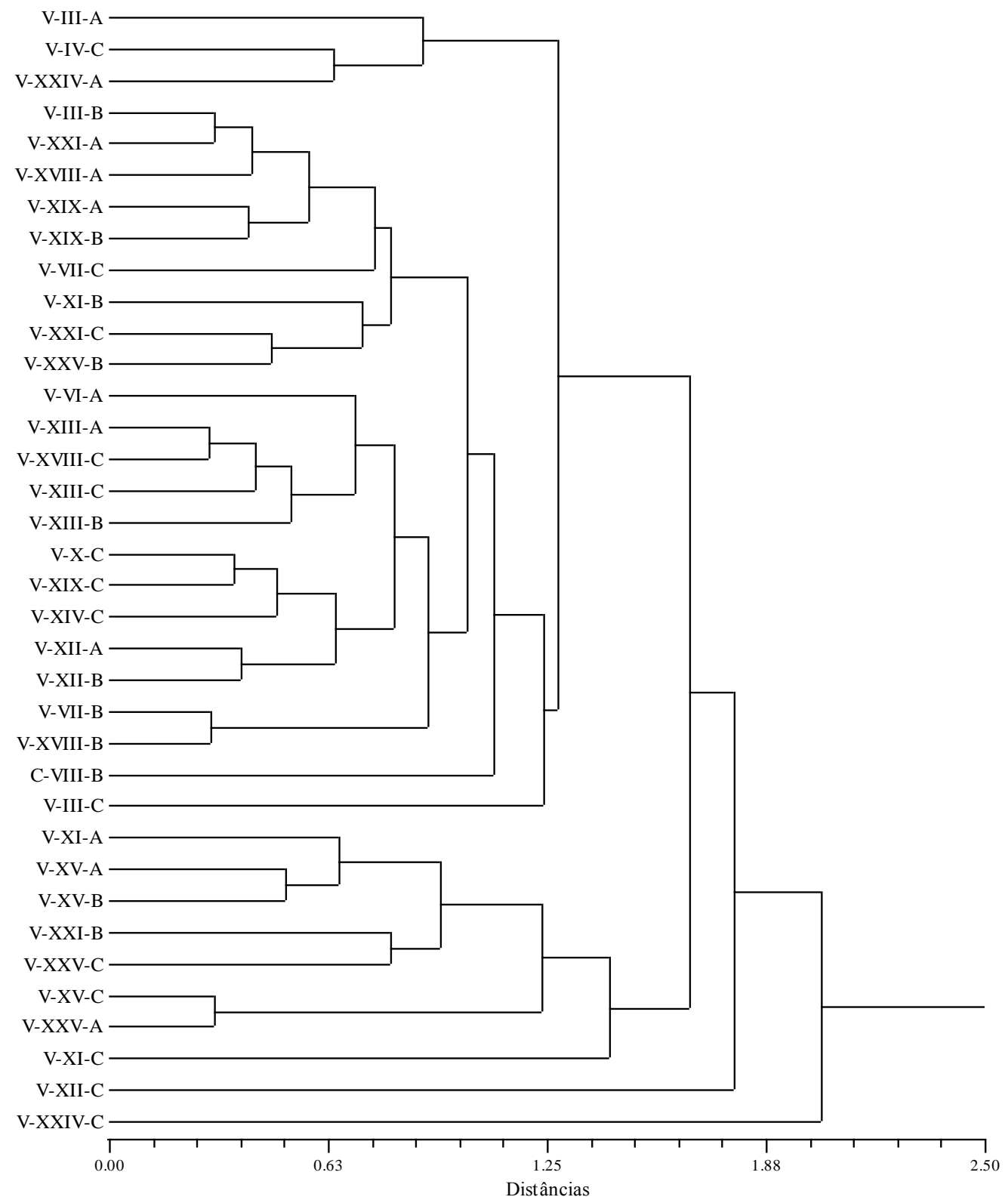

Figura 4. Fenograma de distância das 36 amostras, baseado no método UPGMA, aplicado à matriz distâncias $(\mathrm{r}=0,798)$.

Figure 4. Phenogram distance of 36 samples, based on the UPGMA method, applied to matrix distances $(\mathrm{r}=0,798)$. 


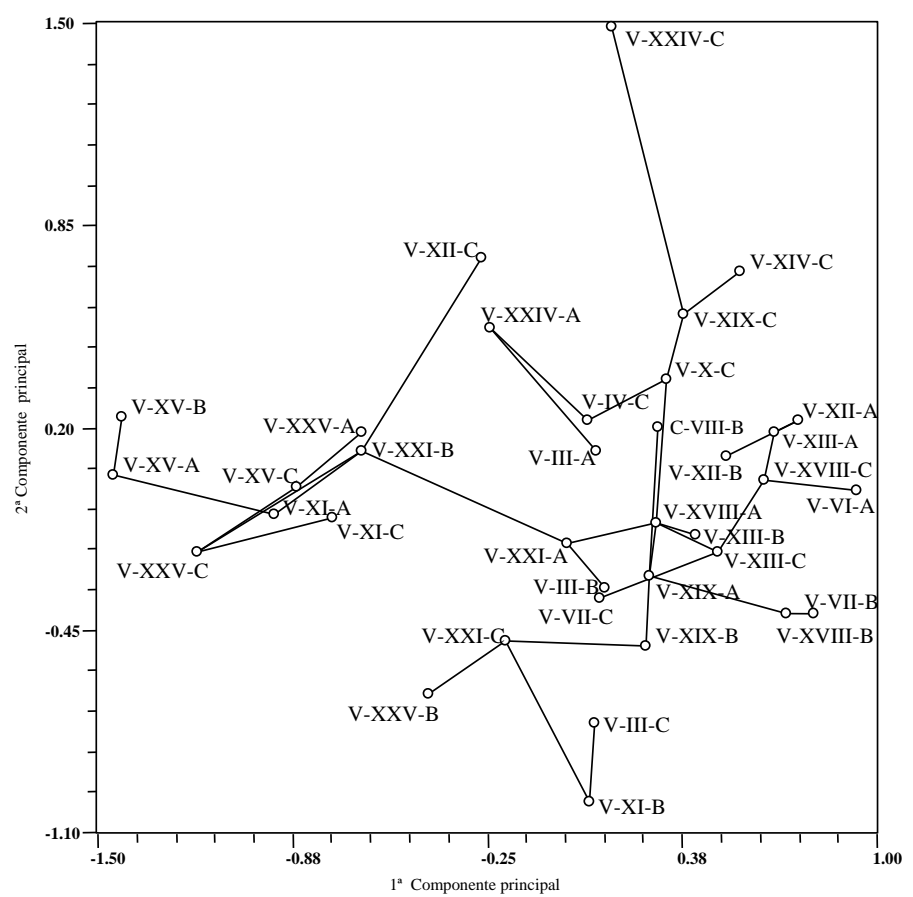

Figura 5. Projeção de 36 amostras no plano definido pelas duas primeiras componentes principais, a que foi sobreposta a árvore de conexão mínima para a detecção de distorções locais.

Figure 5. Projection of 36 samples in the plane of the first two principal components, which was superimposed on the minimum spanning tree to the detection of local distortions.

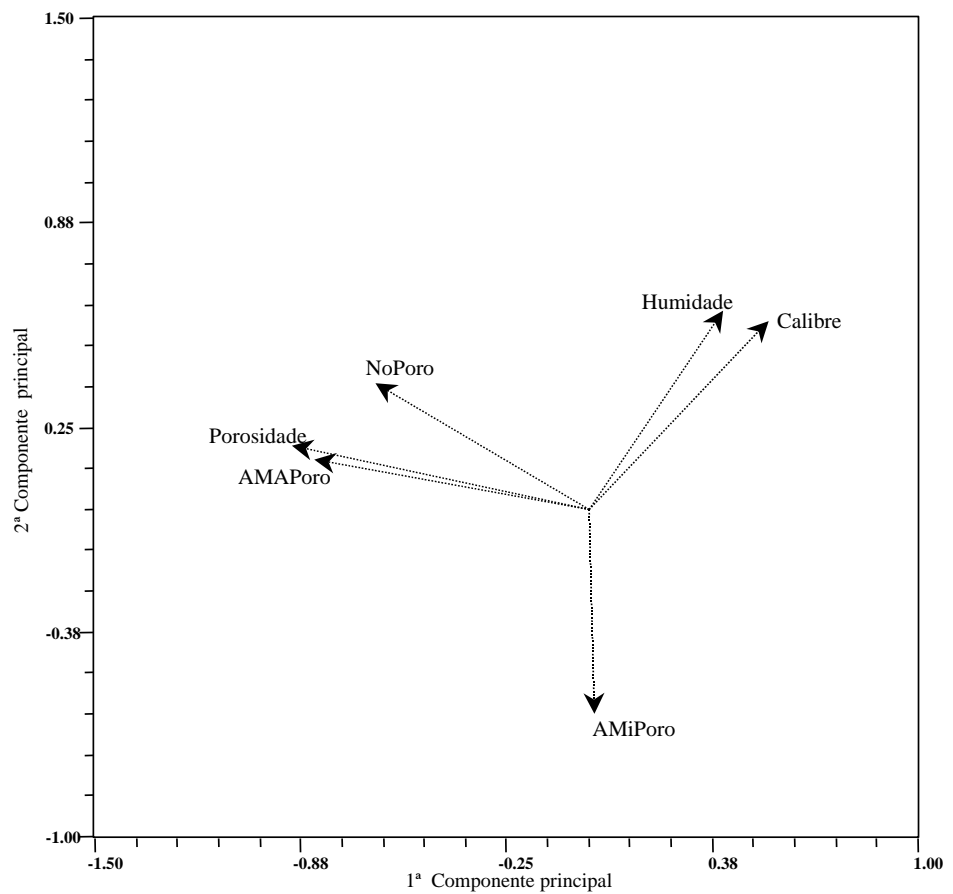

Figura 6. Projeções das variáveis físicas no plano definido pelas duas primeiras componentes principais. Figure 6. Projections of the physical variables in the plane of the first two principal components. 


\section{CONCLUSÕES}

- A classificação proposta e validada foi baseada nas características físicas e na análise de imagem, a qual é consistente e objetiva, tendo repetitividade assegurada, o que não se verifica com o critério de pontuação (avaliação visual).

- Conclui-se que a característica com maior peso é o calibre, seguida da porosidade, ambas características seletivas das cortiças para o fabrico de rolhas, sendo depois a força correspondente ao binário máximo e o respectivo ângulo.

- As cortiças utilizadas para a produção de rolha classificadas de boas apresentam baixa porosidade, mas um ângulo de torção e força correspondente ao binário máximo altos. Portanto, conclui-se que o ângulo médio, a área mínima de poros e a força média são inversamente proporcionais em relação ao número de poros, área máxima de poros e porosidade.

- Pelos critérios apresentados, algumas amostras inicialmente não se destinavam à produção de rolhas, mas tiveram esse destino, como foi o caso das amostras C-IV-B e C-IV-A, em que a escolha se verificou acertada por esse método.

- No conjunto das cortiças não utilizadas para rolhas, podem-se encontrar amostras que inicialmente dariam origem a rolhas, mas a sua não utilização para a fabricação das mesmas deveu-se sobretudo aos resultados obtidos pela análise de imagem, uma vez que indicavam valores de porosidade elevados (por exemplo: a amostra V-IV-C, pelo valor do seu calibre, 29,33 mm, é considerada meiamarca, no entanto não foi utilizada para fabrico de rolhas, pois apresenta uma porosidade de 3,4\%).

- Conclui-se também, pelo conjunto de resultados, em favor da utilidade da análise de imagem e do ensaio de torção como técnicas expeditas na caracterização da cortiça destinadas à produção de rolha, permitindo assim a validação do método de classificação aplicada ao trabalho realizado por Pestana da Silva (2003).

\section{REFERÊNCIAS}

CARNEIRO, L. C. Aplicação de métodos de taxonomia numérica à ampelografia de Vitis vinifera $\mathbf{L}$. Tese de Doutoramento. ISA, Universidade Técnica de Lisboa, 1987.

FORTES, M. A.; ROSA, M. E.; PEREIRA, H. A cortiça. IST Press, Lisboa, 2004.

PESTANA, M.; LOUZADA, J.; SARDINHA, R. Classificação das cortiças para a produção de rolha, recorrendo a um critério objectivo. Parte I - As Rabanadas, Silva Lusitana, Lisboa, v. 13, n. 1, p. 51-73, 2005.

. Classificação das cortiças para a produção de rolha, recorrendo a um critério objectivo. Parte III As Rolhas, Silva Lusitana, Lisboa, v. 14, n. 1, p. 35-49, 2006.

PESTANA DA SILVA, M. A caracterização da qualidade da cortiça para a produção de rolha: aplicação da metodologia de análise multivariada com vista à validação de grupos de qualidade. Tese de Doutoramento, Vila Real, 2003.

REIS, E. Estatística multivariada aplicada. 2. Ed. Lisboa: Edições Sílabo, 2001.

ROHLF. NTSYSPC Ver. 2.1 (Numerical Taxonomy and Multivariate Analysis System), Exeter Software. Setanket. New York, 1997.

SNEATH, P. H. A.; SOKAL, R. R.. Numerical Taxonomy. The principles and practice of numerical classification. W. F. Freeman, San Francisco, 1973. (Cit. CARNEIRO, 1987).

SOKAL, R. R.; ROHLF, F. J. The comparation of dendograms by objective methods. Taxon, v. 11, p. 33-40, 1962. (Cit. CARNEIRO, 1987). 